\title{
Thoughts on One Thousand Chronic Cough Patients
}

\author{
Peter V. Dicpinigaitis
}

Received: 6 June 2012/ Accepted: 12 September 2012/Published online: 27 September 2012

(C) Springer Science+Business Media, LLC 2012

\begin{abstract}
Cough is the most common complaint for which patients seek medical attention. Although most cases of acute cough, due to viral upper respiratory tract infection, are transient and self-limited, chronic cough often poses a diagnostic and therapeutic challenge. A subgroup of patients suffering from chronic cough will elude a diagnosis despite appropriate evaluation, and will prove refractory to all therapeutic intervention. Having personally evaluated 1000 individuals presenting to a specialty cough center, the author shares insights regarding the demographic composition of this unique group of patients, as well as clinical observations and opinions on currently unmet needs requiring further research.
\end{abstract}

\section{Introduction}

Cough is the most common complaint for which patients in the United States seek medical attention [1]. Thankfully, most episodes of cough result from an acute viral upper respiratory tract infection and are transient and self-limited. However, a significant subgroup of adult patients with chronic cough (defined as cough of greater than 8 weeks' duration) eludes the establishment of a definitive diagnosis and fails to respond to a series of diagnostic-therapeutic trials of medication addressing the major etiologies of chronic cough: upper airway cough syndrome (formerly postnasal drip syndrome); eosinophilic airway inflammation; and gastroesophageal reflux disease (GERD) [2]. The frequently unsuccessful effort to treat refractory cough represents a huge source of frustration for

P. V. Dicpinigaitis $(\square)$

Albert Einstein College of Medicine and Montefiore Medical

Center, 1825 Eastchester Road, Bronx, NY 10461, USA

e-mail: pdicpinigaitis@pol.net patient and physician alike. Indeed, the significance of chronic cough as a clinical issue is clearly demonstrated by the fact that, in the past decade, no fewer than six major respiratory societies worldwide have published guidelines on the diagnosis and management of chronic cough [2-7].

To address the challenges presented by this particularly difficult population, the Montefiore Cough Center (Bronx, NY) was established in 2003. Recently, the Cough Center welcomed its 1,000 th new patient. Having evaluated each patient personally, the author has gained considerable insight into this unique, fascinating, and complex population. Alas, the many instances of patient exhilaration and physician satisfaction resulting from therapeutic successes continue to be balanced by the shared frustration of cases of severe, relentless cough refractory to all intervention. Thus, our recently attained milestone has motivated the author to share some of the observations made, lessons learned, and obstacles not yet overcome, in the course of evaluating and managing our first 1,000 chronic cough patients.

My goals in this discourse are to share clinical information that may be useful to my colleagues who struggle with similar challenging patients, as well as to raise questions, concerns, and highlight areas of unmet need that, hopefully, may stimulate clinical investigators, basic scientists, and the pharmaceutical industry to initiate or accelerate their research efforts in the field of cough mechanisms and therapeutics. To provide some structure to my list of comments and observations, I have chosen the subdivisions of demographics, clinical observations, and need for future research.

\section{Demographics}

1. A gender difference in cough has clearly been established. Healthy women have a more sensitive cough 
reflex than do healthy men $[8,9]$, and women with chronic cough demonstrate an enhanced cough reflex compared with their male counterparts [10]. In terms of health-related quality of life, women are significantly more adversely affected by chronic cough than are men [11]. Our experience confirms that of other chronic cough centers in that female patients predominate; $69.7 \%$ of our first 1,000 patients evaluated were women.

2. Little attention has been paid to age as regards chronic cough. The recently emerged profile of a "typical" refractory chronic cough patient has been that of a perimenopausal woman, often recollecting the onset of cough coincident with a distant upper respiratory tract infection [12]. Thus, we found it noteworthy that elderly patients were significantly represented in our population: $34.6 \%$ age 65 years and older; $23.9 \%$ age 70 years and older. This is an interesting observation in light of the fact that what little work has been done with elderly patients has demonstrated a decrease in cough reflex sensitivity with aging, thus offering an explanation for the increased incidence of aspiration in this population [13]. Whether microaspiration or an increased incidence of GERD may explain the prevalence of chronic cough among the elderly remains unknown.

3. The vast majority of patients presenting for evaluation of chronic cough are lifetime nonsmokers. Indeed, only $2.7 \%$ of patients we have evaluated were active smokers and $27 \%$ former smokers. A possible explanation for this observation is that smokers may expect a cough as a result of their habit, and thus are reluctant or not motivated to seek evaluation. Others who have been diagnosed with chronic obstructive pulmonary disease may assume that their cough continues despite therapy for obstructive lung disease, without considering that other etiologies of cough may be present.

4. With each passing year, a greater percentage of our patients presents to us as self-referrals, rather than having been referred by a physician. The most common report is that the patient found the cough center through an internet search, with many coming great distances for evaluation. Often, concerned family members, friends, or coworkers performed the research on the patient's behalf. A very typical history describes the patient having been seen by numerous physicians, including various subspecialists, over the course of many years, without success, and either being dismissed from further evaluation and treatment or themselves yielding to frustration and abandoning further efforts until learning of the existence of a specialty cough center. Most of these patients are highly motivated and desperate for further assistance and guidance.

\section{Clinical Observations}

5. In the 2006 guidelines for the diagnosis and management of cough published by the American College of Chest Physicians (ACCP) [2], the term postnasal drip syndrome was abandoned in favor of a new, more descriptive and inclusive term: upper airway cough syndrome (UACS). UACS describes chronic cough in association with one or more of the following symptoms: the sensation of material dripping down the back of the throat; nasal discharge; and frequent throat clearing. The new terminology addressed the uncertainty of whether cough was due specifically to the postnasal drip or whether, in some instances, irritation or inflammation of upper airway structures could directly stimulate cough receptors and cause cough independently of, or in addition to, postnasal drip [2]. Because UACS is believed to be one of the most common etiologies of chronic cough, a trial of therapy with a first-generation antihistamine (with or without a decongestant) is a standard early step in the diagnostic-therapeutic protocol recommended in the guidelines of the ACCP and others [2]. Indeed, in a subgroup of patients, a 2-week course of therapy will lead to cough resolution and a presumptive diagnosis of UACS as the sole cause of cough will have been established. However, in a remarkable number of patients, we have witnessed one of three alternative patterns. In one group, the initial course of therapy with a first-generation antihistamine leads to symptom resolution, but cough eventually recurs (at varying intervals), necessitating subsequent courses of therapy that are similarly effective. In another group, the initial course of therapy leads to resolution of cough, but when the medication is repeated after cough recurrence, little or no improvement results. Yet another group initially experiences transient resolution or significant improvement in cough, but the cough then worsens while the 2-week course of empiric therapy is still ongoing. Such patterns of response might suggest that the first-generation antihistamines are acting purely as antitussives in these patients, rather than as medications ameliorating underlying UACS. One possibility is that these agents are suppressing cough not through their action as antihistaminics, but as centrally penetrating anticholinergics [14]. Alternatively, recent investigation has suggested that first-generation antihistamines may be acting as antagonists of the TRPV1 receptor, the significance of which in the mechanism of cough has been recently documented [15-17].

6. Many patients present for evaluation with the misperception that the main etiologies of chronic cough have 
been excluded in their case, simply because they had previously undergone therapeutic regimens that were in fact inadequate to reach such a conclusion. Common examples would be a course of therapy with a newergeneration, nonsedating antihistamine (loratadine, cetirizine, fexofenadine) in the case of UACS; treatment limited to inhaled rather than oral corticosteroids in the case of asthma or nonasthmatic eosinophilic bronchitis (EB);[18] and, regimens for GERD that did not include at least 2 months' therapy with a proton-pump inhibitor administered twice daily, in association with strict adherence to an antireflux diet and lifestyle measures. The addition of a prokinetic agent, such as metoclopramide, should be considered after 2 months of such a regimen in the absence of an alternative diagnosis. Thus, perhaps the most important role of the physician evaluating a patient with chronic cough is to thoroughly review previous treatment regimens and to ascertain that appropriate components of the diagnostic-therapeutic protocol are administered [2].

7. A particularly frustrating group of patients is comprised of those who achieve complete cough resolution after a short course of oral steroids (i.e., $40 \mathrm{mg}$ daily for 5 days, followed by $20 \mathrm{mg}$ for 5 days), yet experience recurrence of cough back to baseline despite a regimen of inhaled corticosteroids (ICS) and a leukotriene-receptor antagonist [2, 18]. In such patients with cough-variant asthma or EB, therapy exclusive of systemic steroids should be optimized by meticulous attention to ICS compliance and inhaler technique, environmental factors, etc. Measurement of exhaled nitric oxide can support the use of antiinflammatory therapy, as the presence of elevated exhaled nitric oxide levels is predictive of a favorable response to ICS [19].

8. We still regularly see patients referred for evaluation of chronic cough, who continue to be maintained on angiotensin-converting enzyme (ACE) inhibitors because their cough predated the initiation of ACE inhibitor therapy. It is essential to discontinue the ACE inhibitor regardless of the temporal relation to cough onset, because ACE inhibitor administration enhances cough reflex sensitivity and may be potentiating another underlying etiology of chronic cough [20].

9. Psychogenic cough is a well-documented entity in the pediatric population but is uncommon in adults [21]. In our opinion, a diagnosis of psychogenic cough should be made with great caution, because the result would likely be a cessation of further investigation of other etiologies of cough. After evaluation of 1,000 patients at our cough center, we have yet to make a diagnosis of psychogenic cough.

\section{Need for Future Research}

10. The treatment of cough presumed due to GERD continues to pose a great challenge. In the past decade, it has become clear that a significant percentage of patients may have cough due to nonacid reflux, thus explaining the failure of aggressive antacid therapy [22]. Hence, in patients with cough presumed due to reflux but refractory to intensive acid-suppression therapy and lifestyle measures, the addition of a prokinetic agent is indicated, and, in our experience, often effective. Unfortunately, after the removal from the market of cisapride because of complications, including ventricular arrhythmias, the only prokinetic agent available in the United States is metoclopramide, a drug also associated with intolerable side effects [23]. Thus, a safe and effective prokinetic agent represents a great unmet need at present.

11. As mentioned above, because a significant number of patients suffer chronic cough due to nonacid reflux, a standard evaluation with endoscopy and 24-h esophageal pH testing, previously considered a "gold standard," would be expected often to be inadequate. Indeed, recent investigation has demonstrated a higher diagnostic yield when 24-h, multichannel, intraluminal, pH-impedance studies, which document both acid and nonacid reflux events, are performed [24]. Thus, prospective studies would be welcome to provide further insight into the true value of this diagnostic modality and to shed light on whether the initial diagnostic test in a patient with chronic cough presumed due to reflux should be a $\mathrm{pH}$-impedance study.

12. The pulmonologist or other subspecialist will no doubt encounter patients whose cough is refractory to all appropriate interventions and empiric therapeutic trials. Although chronic opiate therapy may be helpful [25], such a regimen may not be tolerated or accepted by many patients. Indeed, a safe, effective, nonnarcotic antitussive represents a great unmet need at present. Small studies have described success in such patients with amitriptyline [26] and gabapentin [27]. In our experience, only a very small percentage of patients who have been treated with these agents reported improvement. Further investigation is needed to try to identify the small subgroup of patients who will benefit from such therapeutic interventions.

13. Numerous prospective and observational studies have demonstrated that in the vast majority of nonsmokers not treated with ACE inhibitors and whose chest radiograph does not reveal acute pathology, chronic 
cough will be due to one or more of the following causes: UACS; asthma or EB; and GERD [2]. However, only a very small percentage of individuals with these very common conditions develops chronic cough. Recently, the cough hypersensitivity syndrome [28-30] has been proposed as an explanation for the occurrence of chronic cough in a small subgroup of persons with the same common conditions that do not induce cough in the vast majority of individuals. Further work will be necessary to advance this concept in terms of insight into mechanisms of cough and potential therapeutic strategies.

The patient with chronic cough presents a great diagnostic and therapeutic challenge. The tasks before us include ongoing, meticulous clinical evaluation and management by the informed physician, and acceleration of research efforts by the scientific and pharmaceutical industrial communities in the quest for better, safe, and effective therapies for this common and debilitating condition.

Conflict of interest The author reports no conflict of interest associated with this manuscript.

\section{References}

1. Cherry DK, Hing E, Woodwell DA, Rechsteiner EA (2006) National Ambulatory Medical Care Survey: 2006 summary. Natl Health Stat Rep 3:1-39

2. Irwin RS, Baumann MH, Bolser DC et al (2006) Diagnosis and management of cough executive summary: ACCP evidencebased clinical practice guidelines. Chest 129(Suppl 1):1S-23S

3. Morice AH, Fontana GA, Sovijarvi ARA, Pistolesi M, Chung KF, Widdicombe $\mathbf{J}$ et al (2004) The diagnosis and management of chronic cough. Eur Respir J 24:481-492

4. Morice AH, McGarvey L, Pavord I (2006) British Thoracic Society Cough Guideline Group. Recommendations for the management of cough in adults. Thorax 61(Suppl 1):i1-i24

5. Kohno S, Ishida T, Uchida Y, Kishimoto H, Sasaki H, Shioya T et al (2006) The Japanese Respiratory Society guidelines for management of cough. Respirology 11(Suppl 4):S135-S186

6. Kardos P, Berck H, Fuchs K-H, Gillissen A, Klimek L, Morr H et al (2010) Guidelines of the German Respiratory Society for diagnosis and treatment of adults suffering from acute or chronic cough. Pneumologie 64:336-373

7. Chen MH, Chen P, Zhong NS et al (2011) The Chinese national guidelines on diagnosis and management of cough. Chin Med J (Engl) 124:3207-3219

8. Fujimura M, Kasahara K, Kamio Y et al (1996) Female gender as a determinant of cough threshold to inhaled capsaicin. Eur Respir J 9:1624-1626

9. Dicpinigaitis PV, Rauf K (1998) The influence of gender on cough reflex sensitivity. Chest 113:1319-1321
10. Kastelik JA, Thompson RH, Aziz I et al (2002) Sex-related differences in cough reflex sensitivity in patients with chronic cough. Am J Respir Crit Care Med 166:961-964

11. French CT, Fletcher KE, Irwin RS (2005) A comparison of gender differences in health-related quality of life in acute and chronic coughers. Chest 127:1991-1998

12. McGarvey LP (2008) Does idiopathic cough exist? Lung 186(Suppl 1):S78-S81

13. Ebihara S, Ebihara T, Kohzuki M (2012) Effect of aging on cough and swallowing reflexes: implications for preventing aspiration pneumonia. Lung 190:29-33

14. Bolser DC (2008) Older-generation antihistamines and cough due to upper airway cough syndrome (UACS): efficacy and mechanism. Lung 186(Suppl 1):S74-S77

15. Sadofsky LR, Campi B, Trevisani M, Compton SJ, Morice AH (2008) Transient receptor potential vanilloid-1-mediated calcium responses are inhibited by the alkylamine antihistamines dexbrompheniramine and chlorpheniramine. Exp Lung Res 34:681-693

16. Nassini R, Materazzi S, De Siena G, De Cesaris F, Geppetti P (2010) Transient receptor potential channels as novel drug targets in respiratory diseases. Curr Opin Investig Drugs 11:535-542

17. Adcock JJ (2009) TRPV1 receptors in sensitisation of cough and pain reflexes. Pulm Pharmacol Ther 22:65-70

18. Brightling CE (2010) Cough due to asthma and nonasthmatic eosinophilic bronchitis. Lung 188(Suppl 1):S13-S17

19. Lim KG (2010) Nitric oxide measurement in chronic cough. Lung 188(Suppl 1):S19-S22

20. Dicpinigaitis PV (2006) Angiotensin-converting enzyme inhibitor-induced cough: ACCP evidence-based clinical practice guidelines. Chest 129(Suppl 1):169S-173S

21. Weinberger M (2012) The habit cough syndrome and its variations. Lung 190:45-53

22. Irwin RS, Zawacki JK, Wilson MM, French CT, Callery MP (2002) Chronic cough due to gastroesophageal reflux disease: failure to resolve despite total/near-total elimination of esophageal acid. Chest 121:1132-1140

23. Dicpinigaitis PV (2011) Cough: an unmet clinical need. Br J Pharmacol 163:116-124

24. Lee J-H, Park S-Y, Cho S-B et al (2012) Reflux episode reaching the proximal esophagus are associated with chronic cough. Gut Liver 6:197-202

25. Morice AH, Menon MS, Mulrennan SA, Everett CF, Wright C, Jackson J, Thompson R (2007) Opiate therapy in chronic cough. Am J Respir Crit Care Med 175:312-315

26. Jeyakumar A, Brickman TM, Haben M (2006) Effectiveness of amitriptyline versus cough suppressants in the treatment of chronic cough resulting from postviral vagal neuropathy. Laryngoscope 116:2108-2112

27. Ryan NM, Birring SS, Gibson PG (2012) Gabapentin for refractory chronic cough: a randomised, double-blind, placebocontrolled trial. Lancet [Epub ahead of print]

28. Morice AH, Faruqi S, Wright CE, Thompson R, Bland JM (2011) Cough hypersensitivity syndrome: a distinct clinical entity. Lung 189:73-79

29. Chung KF (2011) Chronic 'cough hypersensitivity syndrome': a more precise label for chronic cough. Pulm Pharmacol Ther 24:267-271

30. Morice AH, McGarvey LPA, Dicpinigaitis PV (2012) Cough hypersensitivity syndrome is an important clinical concept: a pro/ con debate. Lung 190:3-9 